\title{
Depletion Interaction Mediated by a Polydisperse Polymer Studied with Total Internal Reflection Microscopy
}

\author{
Dzina Kleshchanok, Remco Tuinier, and Peter R. Lang* \\ Forschungszentrum Jülich, Institut für Festkörperforschung, Weiche Materie, 52425 Jülich, Germany
}

Received June 9, 2006. In Final Form: August 16, 2006

\begin{abstract}
Total internal reflection microscopy (TIRM) was applied to measure depletion forces between a charged colloidal sphere and a charged solid wall induced by dextran, a nonionic nonadsorbing polydisperse polysaccharide. The polymer size polydispersity is shown to greatly influence the depletion potential. Using the theory for the depletion interaction due to ideal polydisperse polymer chains, we could accurately describe the experimental data with a single adjustable parameter.
\end{abstract}

\section{Introduction}

The depletion interaction between colloidal particles due to nonadsorbing polymers has been studied thoroughly for more than fifty years. Understanding depletion phenomena is relevant for many reasons. First, it helps to get to know when and why phase separation occurs in mixtures of polymers and colloids. ${ }^{1,2}$ Furthermore, depletion-induced phase separation makes it possible to concentrate colloidal dispersions in a convenient way. ${ }^{3,4}$ In addition, if the colloids and/or polymers are polydisperse, depletion-induced phase separation can be used for size fractionation of the components. ${ }^{5,6}$ Besides these practical reasons, depletion studies provide an accessible way of "tuning" the range of the interaction between colloidal particles by varying the diameter of the added nonadsorbing macromolecules, and adjusting the strength of the attraction by changing the concentration of polymers. Colloid-polymer mixtures are therefore model fluids for studying the properties of liquids, as well as crystallization and gelation phenomena. ${ }^{7-9}$

The mechanism that is responsible for the depletion interaction was first explained by Asakura and Oosawa, ${ }^{10}$ and independently by Vrij. ${ }^{11,12}$ It can be understood by regarding two parallel plates at a distance $h$ immersed in a solution of nonadsorbing nonionic polymers, as depicted in Figure 1. There is a concentration gradient in the average equilibrium polymer-segment concentration profiles when going from the bulk (the maximum segment concentration) to the plate surface (where the concentration is zero). A common simplification to calculate the depletion potential is to replace the concentration profiles with a step function. One part of the step function now consists of a layer in which the polymer concentration equals zero, denoted as a depletion

(1) Poon, W. C. K. J. Phys.: Condens. Matter 2002, 14, R859-R880.

(2) Tuinier, R.; Rieger, J.; de Kruif, C. G. Adv. Colloid Interface Sci. 2003 $103,1-31$.

(3) Tuinier, R.; de Kruif, C. G. J. Chem. Phys. 1999, 110, 9296-9304

(4) Tuinier, R.; ten Grotenhuis, E.; Holt, C.; Timmins, P. A.; de Kruif, C. G. Phys. Rev. E 1999, 60, 848-856.

(5) Bibette, J. J. Colloid Interface Sci. 1991, 147, 474-478.

(6) Promislow, J. H. E. Structural Evolution in Magnetorheological Fluids: Kinetics and Energetics. Ph.D. thesis, Stanford University, 1997.

(7) Pham, K. N.; Egelhaaf, S. U.; Pusey, P. N.; Poon, W. C. K. Phys. Rev. E 2004, 69, 011503.

(8) Pham, K. N.; Puertas, A. M.; Bergenholtz, J.; Egelhaaf, S. U.; Moussaid, A.; Pusey, P. N.; Schofield, A. B.; Cates, M. E.; Fuchs, M.; Poon, W. C. K. Science 2002, 296, 104-106.

(9) Poon, W. C. K.; Pirie, A. D.; Pusey, P. N. Faraday Discuss. 1995, 101 $65-76$.

(10) Asakura, S.; Oosawa, F. J. Chem. Phys. 1954, 22, 1255-1256.

(11) de Hek, H.; Vrij, A. J. Colloid Interface Sci. 1981, 84, 409-422.

(12) Vrij, A. Pure Appl. Chem. 1976, 48, 471-483.

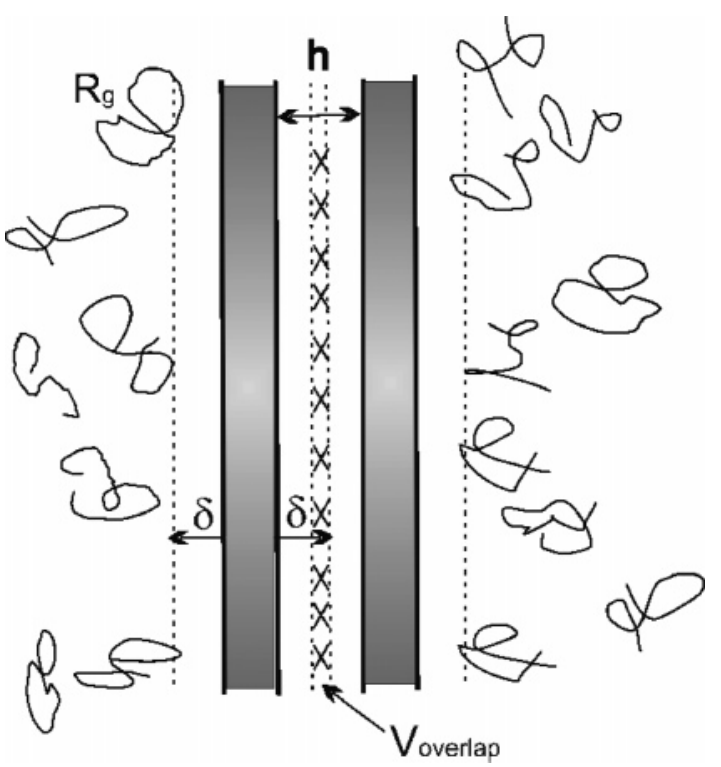

Figure 1. Schematic picture of the depletion zones near two parallel plates in a solution of nonadsorbing polymer chains. The depletion layers are indicated by short dashes. For overlapping depletion layers, shown as hatched area, the osmotic pressure is unbalanced, leading to a net osmotic force that pushes the plates together.

layer with a thickness $\delta$, indicated by the dashed lines along the plate in Figure 1. Outside this layer the polymer concentration equals the bulk polymer concentration. The concentration gradient due to the depletion layer results in an osmotic pressure gradient. For a single plate this osmotic pressure gradient is balanced. However, if the depletion layers of two plates overlap, the osmotic pressure, $\Pi_{p}$, becomes unbalanced leading to a net osmotic force that pushes the plates together. In this case, for ideal polymers, the depletion interaction per unit area equals the product of the overlap volume, $V_{\text {overlap }}=2 \delta-h$ (indicated by the hatched area in Figure 1), and the osmotic pressure, $\Pi_{\mathrm{p}}$. Thus, the depletion potential between two parallel plates can be written as

$$
\phi_{\text {depl,plates }}= \begin{cases}-\Pi_{\mathrm{p}}[2 \delta-h] & \text { for } 0 \leq h \leq 2 \delta \\ 0 & \text { for } h>2 \delta\end{cases}
$$

A large amount of the theoretical work on depletion forces and the resulting phase behavior are based on expressions for the 
pair interaction potentials. ${ }^{13}$ Nowadays it is possible to measure pair interaction potentials directly using optical tweezers, ${ }^{14}$ atomic force microscopy (AFM), ${ }^{15-17}$ and total internal reflection microscopy (TIRM).${ }^{18-23}$ Major advantages of the TIRM technique relative to other direct methods for studying depletion interactions are its extreme sensitivity and its ability to investigate the interactions of a single, freely moving, Brownian particle. The technique allows us to detect potential variations on the order of $k_{\mathrm{B}} T$ and beyond. Several measurements were performed with TIRM on interactions between a sphere and a wall due to different charged depletants (polyelectrolytes, cationic micelles and charged nano-silica particles) ${ }^{18,19,21-23}$ as well as nonadsorbing uncharged polymers. ${ }^{19,20}$ Some of these results are controversial. For example, Pagac et al. ${ }^{19} \mathrm{did}$ not detected any significant depletion interaction between a silica sphere and a glass plate in a solution of noncharged poly(ethylene oxide) (PEO) whereas theoretical results for ideal chains ${ }^{24}$ predict a strong depletion attraction with a contact value of a few hundred $k_{\mathrm{B}} T$ under the experimental conditions Pagac et al applied. Differently, Rudhardt et al. ${ }^{20}$ were able to measure depletion interaction due to the same polymer under conditions where the depletion forces are significantly weaker and the predicted contact value is close to $-10 k_{\mathrm{B}} T$. These controversies might be evoked by the fact that Pagac's measurements were performed under conditions, under which it is not possible to detect depletion forces with TIRM. Therefore, a simple theoretical framework is required to estimate the correct experimental conditions (polymer concentration, particle size and Debye screening length) enabling depletion studies with TIRM.

Another essential issue that has not attracted significant attention in theories and simulations is polydispersity. Because of the kinetics of polymerization, all synthetic and most natural (except for several proteins and viruses) polymers have a finite molar mass distribution. However, in most studies polymers are treated as being monodisperse and incorporation of the size polydispersity of polymers has gained very limited attention in theories for (polymer-induced) depletion. So far, polydisperse polymers were mainly simplified as polydisperse spheres. ${ }^{25-30}$ A first extension toward polydisperse ideal chains was done by Tuinier and Petukhov. ${ }^{31}$ Here we compare their theory with our data on the depletion force induced by polydisperse polymers, as obtained with TIRM.

(13) Gast, A. P.; Hall, C. K.; Russel, W. B. J. Colloid Interface Sci. 1983, 96 , $251-267$.

(14) Verma, R.; Crocker, J. C.; Lubensky, T. C.; Yodh, A. G. Macromolecules 2000, 33, 177-186.

(15) Milling, A.; Biggs, S. J. Colloid Interface Sci. 1995, 170, 604-606.

(16) Piech, M.; Walz, J. Y. J. Colloid Interface Sci. 2002, 253, 117-129.

(17) Wijting, W. K.; Knoben, W.; Besseling, N. A. M.; Leermakers, F. A. M.;

Cohen-Stuart, M. A. Phys. Chem. Chem. Phys. 2004, 6, 4432-4439.

(18) Odiachi, P. C.; Prieve, D. C. Colloids Surf. A 1999, 146, 315-328.

(19) Pagac, E. S.; Tilton, R. D.; Prieve, D. C. Langmuir 1998, 14, 5106-5112.

(20) Rudhardt, D.; Bechinger, C.; Leiderer, P. J. Phys.: Condens. Matter 1999, 11, 10073-10078.

(21) Sharma, A.; Tan, S. N.; Walz, J. Y. J. Colloid Interface Sci. 1997, 191, 236-246.

(22) Sharma, A.; Walz, J. Y. J. Chem. Soc., Faraday Trans. 1996, 92, 49975004

(23) Sober, D. L.; Walz, J. Y. Langmuir 1995, 11, 2352-2356.

(24) Bringer, A.; Eisenriegler, E.; Schlesener, F.; Hanke, A. Eur. Phys. J. B 1999, 11, 101-119.

(25) Piech, M.; Walz, J. Y. J. Colloid Interface Sci. 2000, 225, 134-146. (26) Chu, X. L.; Nikolov, A. D.; Wasan, D. T. Langmuir 1996, 12, 50045010.

(27) Goulding, D.; Hansen, J.-P. Mol. Phys. 2001, 99, 865-874

(28) Sear, R. P.; Frenkel, D. Phys. Rev. E. 1997, 55, 1677-1681.

(29) Walz, J. Y. J. Colloid Interface Sci. 1996, 178, 505-513.

(30) Warren, P. B. Langmuir 1997, 13, 4588-4594. 984.
The paper is organized as follows: in section II we first give a simple theoretical prediction of the conditions under which the depletion interaction between a colloidal particle and a wall is measurable with TIRM, followed by a brief review of the theory of depletion effects mediated by ideal polydisperse polymers, whereas in section III we present our experimental system and TIRM-equipment. Experimental findings are reported and comparisons of theory and experiment are discussed in section IV. Finally, we give short conclusions in section V.

\section{Theory}

II.A. Conditions under Which the Depletion Interaction is Measurable with TIRM. In aqueous solutions a colloidal sphere that is denser than water sediments toward the bottom wall of the container. If their surfaces are like charged, the particle will experience an electrostatic repulsion from the wall at distance $h$, which now is the closest distance between the wall and the sphere surface. The superposition of gravitation and electrostatic repulsion leads to an interaction potential, $\phi_{\text {tot }}(h)$, which has a shallow minimum at a separation distance $h_{\text {min }}^{0}$. Due to Brownian motion, however, the particle position will not be fixed at this equilibrium distance. It will rather sample a distribution of heights, $p(h)$, which is related by Boltzmann's equation to the interaction potential $\phi_{\mathrm{tot}}(h)$ :

$$
p(h)=A \exp \left(\frac{-\phi_{\mathrm{tot}}(h)}{k_{\mathrm{B}} T}\right)
$$

where $A$ is a constant normalizing the integrated distribution to unity.

The particle height fluctuations resulting from the thermal motion can be directly observed by TIRM. This technique exploits the properties of the evanescent wave which is formed when a laser beam undergoes total reflection at an optical interface. ${ }^{32-34}$

To calculate the total interaction between a sphere and a plate in a polymer solution, we assume three contributions to the potential which are gravitational energy $\phi_{\mathrm{G}}(h)$, electrostatic repulsion $\phi_{\mathrm{el}}(h)$, and depletion $\phi_{\mathrm{depl}}(h)$. When the separation distance is larger than the range of the van der Waals attraction, the latter is negligible. Thus, in the superposition approximation we write:

$$
\phi_{\mathrm{tot}}(h)=\phi_{\mathrm{el}}(h)+\phi_{\mathrm{depl}}(h)+\phi_{\mathrm{G}}(h)
$$

In the linear Poisson-Boltzmann regime the electrostatic pair interaction between two like charged plates immersed in electrolyte solution with a dielectric constant $\epsilon$, reads ${ }^{35}$

$$
\frac{\phi_{\text {el,plates }}(h)}{k_{\mathrm{B}} T}=2 \epsilon_{0} \epsilon_{\mathrm{r}} \psi_{0}^{2} \kappa \exp (-\kappa h)
$$

This holds for constant Stern potential of the plates, $\psi_{0}$, that is given by their surface charge density, $\sigma$, as $\psi_{0}=\sigma / \epsilon \epsilon_{0} \kappa$, where $\kappa^{-1}$ is the Debye screening length, which is related to the electrolyte concentration of the solution, $c_{\mathrm{el}}$, by $\kappa^{-1}[\mathrm{~nm}]=$ $0.304 \sqrt{c_{\mathrm{el}}}[\mathrm{mol} / \mathrm{L}]^{35}$ for $1-1$ electrolytes in water. To compare predicted interaction potentials with the experimental TIRM data, we calculated potentials between a curved surface (sphere) and a plate by means of the Derjaguin approximation: ${ }^{35}$

(32) Bike, S. G. Cur. Opin. Colloid Interface Sci. 2000, 5, 144-150.

(33) Prieve, D. C. Adv. Colloid Interface Sci. 1999, 82, 93-125.

(34) Walz, J. Y. Cur. Opin. Colloid Interface Sci. 1997, 2, 600-606.

(35) Israelachvili, J. N. Intermolecular and Surface Forces, 2nd ed.; Academic Press: London, 1991. 


$$
\phi_{\text {sphere-plate }}(h)=-2 \pi a \int_{h}^{\infty} \phi_{\text {plate-plate }}\left(h^{\prime}\right) \mathrm{d} h^{\prime}
$$

which holds if the radius of the sphere, $a$, is large as compared to the range of the potential. Because the ranges of the electrostatic repulsion and depletion-induced attraction are smaller than 100 $\mathrm{nm}$ in our experiments and the radius of the sphere we used is close to $3 \mu \mathrm{m}$, this approximation is justified for our measurements. Thus, the electrostatic potential between a charged sphere and a charged plate reads:

$$
\frac{\phi_{\text {el,sphere-plate }}(h)}{k_{\mathrm{B}} T}=4 \pi a \epsilon_{0} \epsilon_{\mathrm{r}} \psi_{0}^{2} \exp (-\kappa h)=B \exp (-\kappa h)
$$

It is not required to determine the amplitude $B$ of the exponential in eq 6 explicitly, because it is related to $h_{\min }^{0}$ by $\kappa h_{\min }^{0}=\ln (\kappa B / G)$ in the absence of depletion forces. ${ }^{33}$ The gravitational contribution to the total potential is given by

$$
\phi_{G}(h)=G h
$$

where $G=(4 / 3) \pi a^{3} \Delta \rho g$ is the buoyancy-corrected net weight of the sphere, with $\Delta \rho$ the particles excess mass density and $g$ the acceleration of gravity. Because we can independently measure $\phi_{G}(h)$ and $\phi_{\mathrm{el}}(h)$, the depletion potential $\phi_{\mathrm{depl}}(h)$ can be calculated from the measured $\phi_{\text {tot }}(h)$, see eq 3 .

Applying the Derjaguin approximation for eq 1 gives the following expression for the depletion attraction between a sphere and a plate:

$$
\begin{aligned}
& \phi_{\text {depl,sphere-plate }}(h)= \\
& \begin{cases}-2 \pi a \Pi_{\mathrm{p}} \int_{h}^{2 \delta}\left[2 \delta-h^{\prime}\right] \mathrm{d} h^{\prime} & \text { for } 0 \leq h \leq 2 \delta \\
0 & \text { for } h>2 \delta\end{cases}
\end{aligned}
$$

In the paper of $\mathrm{Vrij}^{12}$ a simplification of an ideal polymer chain by replacing it with a penetrable hard sphere (PHS) is proposed. A PHS is a sphere that is hard for a colloidal particle, but which can freely permeate through another PHS. For the radius of a PHS one should take the depletion thickness $\delta$. A calculation of the depletion thickness, $\delta$, from the segment density profile of ideal chains near a flat wall made by Eisenriegler ${ }^{36}$ also gives ${ }^{37}$

$$
\delta=\frac{2 R_{\mathrm{g}}}{\sqrt{\pi}}
$$

After integration of $2 \delta-h^{\prime}$ in eq 8 , using $\delta=2 R_{\mathrm{g}} / \sqrt{\pi}$, and substituting the osmotic pressure, $\Pi_{\mathrm{p}}$, in the dilute polymer limit by $\Pi_{\mathrm{p}} / k_{\mathrm{B}} T=\rho_{\mathrm{p}}=\left(c / c^{*}\right)\left(3 / 4 \pi R_{\mathrm{g}}{ }^{3}\right)$, we acquire a simple analytical expression for the depletion potential between a sphere and a plate:

$$
\begin{aligned}
& \frac{\phi_{\text {depl,sphere-plate }}(h)}{k_{\mathrm{B}} T}= \\
& \begin{cases}-\frac{a}{R_{\mathrm{g}}} \frac{c_{\mathrm{p}}}{c^{*}}\left[3\left(\frac{\delta}{R_{\mathrm{g}}}\right)^{2}-3\left(\frac{\delta}{R_{\mathrm{g}}}\right)\left(\frac{h}{R_{\mathrm{g}}}\right)+\frac{3}{4}\left(\frac{h}{R_{\mathrm{g}}}\right)^{2}\right] & \text { for } 0 \leq h \leq 2 \delta \\
0 & \text { for } h>2 \delta\end{cases}
\end{aligned}
$$

Here $c_{\mathrm{p}}$ is the polymer mass concentration in $\mathrm{g} / \mathrm{L}$, the polymer overlap concentration $c^{*}$ is related to the molar mass $M$ and the

(36) Eisenriegler, E. J. Chem. Phys. 1983, 79, 1052-1064

(37) Tuinier, R.; Vliegenthart, G. A.; Lekkerkerker, H. N. W. J. Chem Phys. 2000, 113, 10768-10775.

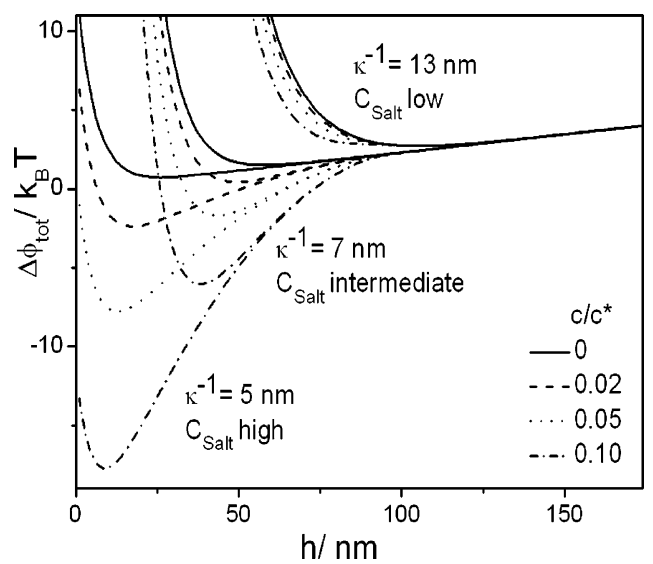

Figure 2. Total interaction potential, $\Delta \phi_{\mathrm{tot}}(h)$, between a charged $5.7 \mu \mathrm{m}$ diameter particle and a charged wall at various Debye lengths. The curves were calculated using eq $3,6,7$, and 10 for a solution containing polymer chains with a radius of gyration of $44 \mathrm{~nm}$. The polymer concentrations of the solutions are indicated in the legend.

radius of gyration $R_{\mathrm{g}}$ of the polymer as $c^{*}=3 M / 4 \pi R_{\mathrm{g}}{ }^{3} N_{\mathrm{A}}$. As follows from eq 10 at fixed size ratio $a / R_{\mathrm{g}}$, an increase of the polymer concentration, $c_{\mathrm{p}}$, leads to a proportional increase of the magnitude of the attraction between the plate and the sphere. A decrease of the size ratio $a / R_{\mathrm{g}}$ at fixed polymer concentration leads to a decrease of the contact potential and an increase of the range of attraction. Thus, it is possible to "tune" the range of the depletion interaction by varying $R_{\mathrm{g}}$ of the macromolecules; the interaction strength can be varied by changing the polymer concentration.

Now, when all the contributions $\left(\phi_{\mathrm{G}}(h), \phi_{\mathrm{el}}(h), \phi_{\mathrm{depl}}(h)\right)$ to the total interaction potential, $\phi_{\text {tot }}(h)$, are known, we can calculate it for different experimental conditions by inserting eq 6,7 , and 10 into eq 3.

In Figure 2 we demonstrate the influence of polymer and salt concentration on the predicted total potential for a size ratio $a / R_{\mathrm{g}}$ $\sim 70$ and $R_{\mathrm{g}}=44 \mathrm{~nm}$. In the low salt concentration regime at $\kappa^{-1}=13.0 \mathrm{~nm}$ without any polymer present, the equilibrium distance of the particle from the surface, $h_{\text {min }}^{0}$, exceeds two depletion thicknesses. Under these conditions the contribution of the depletion interaction is not sufficient to significantly affect the total potential, $\phi_{\text {tot }}(h)$. In this case the potential profiles do not perceptibly change with increasing polymer concentration. An increase of the salt concentration leads to higher screening of the electrostatic repulsion, $\kappa^{-1}=7.6 \mathrm{~nm}$, which reduces $h_{\min }^{0}$. The total potential now deepens with increasing polymer concentration and the depletion interaction should be clearly observable. At even larger salt concentration, $\kappa^{-1}=5.6 \mathrm{~nm}$, the depletion interaction has a very pronounced influence on $\phi_{\text {tot }}(h)$. However, it is not experimentally accessible, because $h_{\min }^{0}$ is now in a range where van der Waals attraction between the sphere and the plate will dominate the potential.

II.B. Depletion Interaction Mediated by Polydisperse Ideal Chains. In section II.A we gave simple predictions for the total potential on the basis of PHS and step function approximations for the depletion interactions. That makes it possible to estimate required experimental conditions for a depletion attraction measurement with TIRM. To enable a quantitative comparison between experimental TIRM data and theory, we now present exact expressions for the depletion interaction between a sphere and a wall due to ideal polymer chains.

Asakura and Oosawa ${ }^{10}$ calculated the force between two plates immersed in a solution of nonadsorbing uncharged monodisperse polymers. This was the first theory on depletion interaction due 
to ideal polymer chains. Using statistical mechanics, they derived an expression for the partition coefficient, $\chi$, which is the polymer concentration between the plates divided by the concentration outside the plates. The partition coefficient allows us to calculate the osmotic pressure difference between the plates as a function of the separation distance, $h$. Integration of this force then yields the depletion interaction potential: ${ }^{36,37}$

$$
\frac{\phi_{\text {depl,plates }}(h)}{\rho_{\mathrm{p}} k_{\mathrm{B}} T}=-\chi h+h-2 \delta
$$

where the proper boundary condition $\phi_{\text {depl,plates }}(\infty)=0$ was used, recovering the depletion layer thickness per plate, $\delta=2 R_{\mathrm{g}} / \sqrt{ } \pi$; $\rho_{\mathrm{p}}$ is the number of polymer chains per volume.

For two plates immersed in a solution of polydisperse polymers the same analysis was performed by Tuinier and Petukhov, ${ }^{31}$ also leading to eq 11, but with different polydispersity-dependent functions $\chi$ and $\delta$. The polydisperse partition coefficient, $\chi_{\text {poly }}$, reads

$$
\chi_{\text {poly }}=\frac{\int_{0}^{\infty} \chi(h, M) \Psi(M) \mathrm{d} M}{\int_{0}^{\infty} \Psi(M) \mathrm{d} M}
$$

which replaces $\chi$ in eq 11. $\Psi(M)$ is the weight distribution of the polymer molar mass, which further will be called the molar mass distribution for convinience. $\chi(h, M)$ is the partition coefficient between two plates due to polymer chains with a molar mass $M$, which is given by

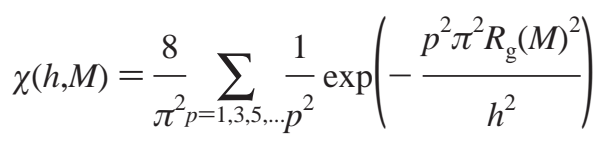

The polydisperse depletion thickness, $\delta_{\text {poly }}$, becomes

$$
\delta_{\text {poly }}=\frac{2}{\sqrt{\pi}} \frac{\int_{0}^{\infty} R_{\mathrm{g}}(M) \Psi(M) \mathrm{d} M}{\int_{0}^{\infty} \Psi(M) \mathrm{d} M}
$$

To relate the polymer concentration $c_{\mathrm{p}}$ (in $\mathrm{g} / \mathrm{L}$ ) to the number of polymer chains per volume $\rho_{\mathrm{p}}$, we use

$$
\rho_{\mathrm{p}}=\frac{c_{\mathrm{p}} N_{\mathrm{A}} \int_{0}^{\infty} \frac{\Psi(M)}{M} \mathrm{~d} M}{\int_{0}^{\infty} \Psi(M) \mathrm{d} M}
$$

Here $N_{\mathrm{A}}$ is Avogadro's number and the concentration $c_{\mathrm{p}}$ reads $c_{\mathrm{p}}=Z \int_{0}^{\infty} \Psi(M) \mathrm{d} M$, with $Z$ being a normalization constant.

The molar-mass-dependent radius of gyration $R_{\mathrm{g}}(M)$, which is required in eqs 13 and 14, is calculated from

$$
R_{\mathrm{g}}(M)=b \sqrt{M}
$$

where the square root corresponds to the ideal polymer chain regime in a $\theta$-solvent. ${ }^{38}$ The pre-factor $b$ depends on the segment length and the chain architecture. Consequently, for a known polymer molar mass distribution, $\Psi(M)$, it is possible to fit the experimental depletion potentials using just one single parameter $b$. Applying the Derjaguin approximation to eq 11 through 15 yields the final expression:

(38) Doi, M. Introduction to Polymer Physics; Clarendon Press: Oxford, U.K., 996.

$$
\frac{\phi_{\text {depl,sphere-plate }}}{\rho_{\text {p,poly }} k_{\mathrm{B}} T}=2 \pi a \int_{h}^{\infty}\left(-\chi_{\text {poly }} z+z-2 \delta_{\text {poly }}\right) \mathrm{d} z
$$

which is required to calculate the interaction between a sphere and a plate due to polydisperse ideal chains. Note that the contact potential between two plates in a solution of polydisperse polymers becomes $-2 \rho_{\text {poly }} k_{\mathrm{B}} T \delta_{\text {poly }}$ for $h \rightarrow 0$.

\section{Experimental Section}

III.A. Samples and Preparation. Polystyrene sulfonate latex spheres with a diameter of $5.7 \mu \mathrm{m}$ (CV 9.5\%) were obtained from Interfacial Dynamics Co., USA. The particles were diluted from the stock suspension down to a volume fraction of $10^{-9}$ for the experiment. The solutions were contained in a carbonized PTFEframe sandwiched between two microscope slides from BK-7 glass, which were received from Fischer Scientific Co., USA. The glass slides were thoroughly cleaned in an ultrasonic bath for $30 \mathrm{~min}$ in $\mathrm{C}_{2} \mathrm{H}_{5} \mathrm{OH}$ before assembling the sample cell.

Dextran with the molar mass distribution $\Psi(M)$, shown in Figure 3 was obtained from Pharmacosmos A/S, Denmark, and used without further purification. The solid line in Figure 3 is a best fit of the molar mass distribution according to a superposition of two Gaussians:

$$
y=\sum_{i=1}^{2} \frac{B_{i}}{\sqrt{0.5 \pi w_{i}}} \exp \left\{-2 \frac{\left(x-x_{c i}\right)_{2}}{w_{i}}\right\}
$$

The best fit values for the parameters $B_{i}$, variance $w_{i}$, and mean $x_{c i}$ are given in Table 1. The $z$-averaged radius of gyration, $\left\langle R_{\mathrm{g}}\right\rangle_{z}=43.7$ $\mathrm{nm}$, and the weight-averaged molar mass of dextran in water, $M_{\mathrm{w}}$ $=2.7 \times 10^{6} \mathrm{~g} / \mathrm{mol}$, were obtained from static light scattering. The measurements were performed with a commercial instrument from ALV-Lasergesellschaft, Germany, equipped with a $15 \mathrm{~mW} \mathrm{HeNe}$ laser at $\lambda_{0}=632.8 \mathrm{~nm}$ from Coherent Inc., USA, as the light source and an avalange diode as the detecting unit. The light scattering data were analyzed using standard procedures. ${ }^{39}$ On the basis of $M_{\mathrm{w}}$ and $\left\langle R_{\mathrm{g}}\right\rangle_{z}$ we roughly estimated $c^{*}=3 M / 4 \pi R_{\mathrm{g}}{ }^{3} N_{\mathrm{A}}$ as $12.4 \mathrm{~g} / \mathrm{L}$. Ultrapure Milli-Q water (resistivity better than $18.2 \mathrm{M} \Omega \mathrm{cm}^{-1}$; Millipore $\mathrm{GmbH}$, Germany) was used as a solvent for all experiments and cleaning steps. Solutions of dextran were prepared by weight. All dextran concentrations used in the measurements were lower than $c^{*}$. Therefore, it was possible to apply the ideal chain approximation. The $\mathrm{pH}$ of samples was adjusted with a standardized stock solution of $0.1 \mathrm{M} \mathrm{NaOH}$ from Aldrich, Germany. All solutions had $\mathrm{pH}=$ 9.7 to prevent the adsorption of dextran on the particle and wall surfaces. Under these conditions $\mathrm{OH}^{-}$-groups replace polymer molecules from the negatively charged surfaces of the latex sphere and the glass. $\mathrm{NaCl}$, ACS grade from Aldrich, Germany, was used to achieve the required Debye length.

III.B. TIRM Measurements. The particle height fluctuations resulting from thermal motion can be directly observed by TIRM. For this purpose a laser beam is directed via a prism to the glass/ solution interface as sketched in Figure 4, with an incident angle $\alpha_{i}$ such that it is totally reflected. The electric field of the laser beam penetrates the interface causing an evanescent wave, the amplitude of which decays exponentially with the distance from the interface. A single colloidal sphere, interacting with this evanescent wave, will scatter the light depending on its position as ${ }^{40}$

$$
I_{\mathrm{s}}(h)=I(h=0) \exp \{-\xi h\}
$$

where $h$ is the distance from the sphere to the wall and $\xi$ is the inverse penetration depth of the evanescent wave. Collecting intensity changes for a sufficiently long period of time provides the probability density of separation distances, which can be converted into a potential energy profile using Boltzmann's equation (eq 2). In a typical TIRM

(39) Brown, W. Light Scattering: Principles and Development; Clarendon Press: Oxford, U.K., 1996.

(40) Prieve, D. C.; Walz, J. Y. Appl. Opt. 1993, 32, 1629-1641. 


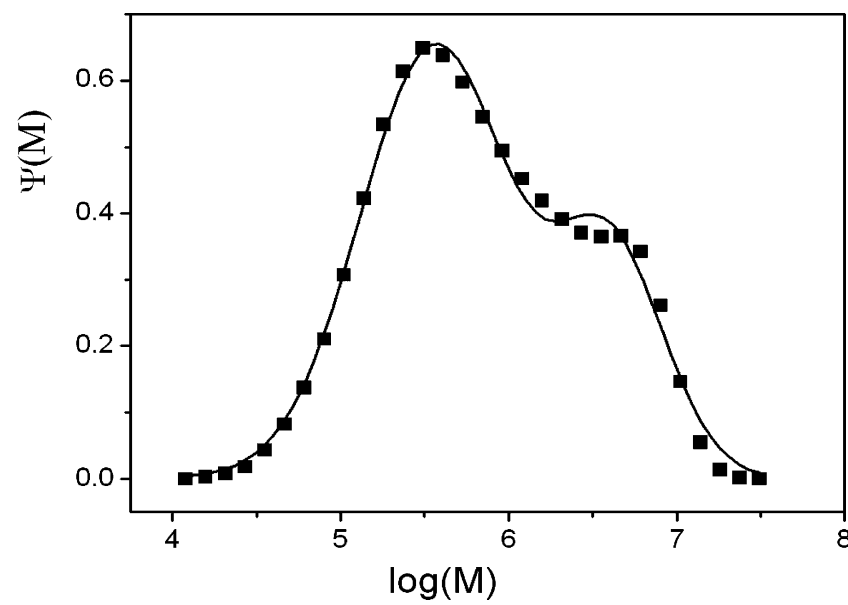

Figure 3. Normalized differential weight fraction distribution of molar masses, $\Psi(M)$, of dextran from Pharmacosmos A/S, Denmark. The solid curve is a best fit according to a distribution consisting of two Gaussians (eq 18).

Table 1. Parameters Used To Fit the Experimental Molar Mass Distribution $\Psi(M)$ of Dextran, Presented in Figure 3

\begin{tabular}{ccc}
\hline$i$ & 1 & 2 \\
\hline$B_{i}$ & 0.732 & 0.275 \\
$w_{i}$ & 0.895 & 0.642 \\
$x_{c i}$ & 5.565 & 6.606
\end{tabular}

experiment static scattering intensities are measured with a time resolution in the range of $10 \mathrm{~ms}$, which translates as $6 \times 10^{4}$ data points recorded for a five minutes measurement.

The experimental setup used was the same as described by Kleshchanok et al. ${ }^{41}$ We used a $15 \mathrm{~mW}$ HeNe laser with $\lambda_{0}=632.8$ $\mathrm{nm}$ to generate the evanescent wave. Additionally, an optical trap was built in to prevent the colloidal particle from moving out of the microscope's observation area. For this purpose a second laser beam was focused directly at the particle from above. We used a Verdi V2 Laser $\left(2 \mathrm{~W}, \lambda_{0}=532 \mathrm{~nm}\right)$ with a tuneable light power output (Coherent Inc., USA) connected to the instrument via an optical fiber from OZ Optics Ltd., Canada. Varying the intensity of the laser beam allows control over the strength of the trap. The trap enabled us to hold the particle while polymer/ electrolyte solutions were replaced by pumping. To allow measurements on one particle under different conditions (salt and polymer concentration), we used a pump (Ecoline VC from Ismatec Laboratoriumstechnik $\mathrm{GmbH}$, Germany) to gently replace the solution while the particle was trapped by the laser. For all experiments we applied an angle of incidence of 63.0 degree, which corresponds to a penetration depth of 220.9 $\mathrm{nm}$ as calculated from the optical path. The exact knowledge of the penetration depth is crucial for the data analysis, because it enters into the conversion of intensities to separation distances. ${ }^{41} \mathrm{~A}$ feasible check is the comparison of the prediction from a superposition of gravitational and electrostatic interaction with the experimental potential from a particle of which the mass and the Debye length is known.

The experimental protocol was as follows: first a potential was obtained in the absence of dextan. Afterward this solution was replaced by a polymer/ electrolyte solution with the same Debye length. After the measurement was performed a new solution with a higher dextran concentration was added. The procedure was repeated until all polymer concentrations were measured. At the end of the experiment a solution with a high salt concentration $(0.1 \mathrm{M} \mathrm{NaCl})$ was pumped to make the particle stick to the surface, to enable the measurement of $I(h=0)$, which is required to convert relative separation distances to absolute values. It was possible to use the same particle to obtain a set of potential profiles for one particular

(41) Kleshchanok, D.; Wong, J. E.; von Klitzing, R.; Lang, P. R. Prog. Colloid Polym Sci. 2006, 133, 52-57.

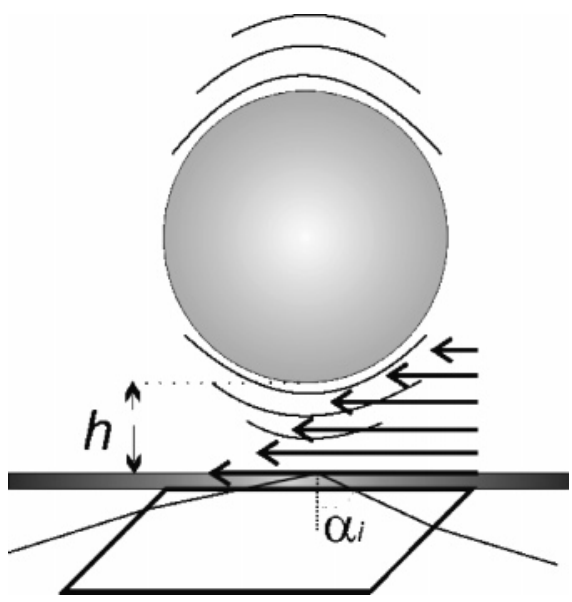

Figure 4. Sketch of the evanescent wave optics. When the incident angle $\alpha_{i}$ is larger than the critical angle, the incident beam is totally reflected at the interface and the evanescent wave penetrates into the fluid. Arrows of different length indicate a decay of the evanescent wave intensity with the distance from the surface. A colloidal particle located close to the surface will scatter light from the evanescent wave. The separation distance between the sphere surface and the reflecting interface is $h$.

Debye length and different polymer concentrations. Thus, a direct comparison between potential profiles was possible.

\section{Results and Discussion}

IV.A. Experimental Findings. Total potentials, $\Delta \phi_{\text {tot }}(h)$, between a $5.7 \mu \mathrm{m}$ diameter polystyrene sphere and a glass wall measured in a solution with the Debye length of $\kappa^{-1}=13.0 \mathrm{~nm}$, are shown in Figure 5. Solid squares show the interaction profile in the absence of dextran. We were able to fit that curve with the superposition of a gravitational contribution and an electrostatic term. According to eqs 6 and 7, and eliminating $B$ from the minimum of $\phi_{\text {tot }}(h)$ the relative potential, $\Delta \phi_{\text {tot }}$, can be obtained in terms of the relative separation distance $h-h_{\min }$ :

$$
\begin{aligned}
& \frac{\Delta \phi_{\mathrm{tot}}\left(h-h_{\min }\right)}{k_{\mathrm{B}} T}=\frac{G}{k_{\mathrm{B}} T}\left(\kappa^{-1}\left[\exp \left\{-\kappa\left(h-h_{\min }\right)\right\}-1\right]+\right. \\
& \left.\left(h-h_{\text {min }}\right)\right)
\end{aligned}
$$

As can be seen from eq 20, the weight of the particle, $G$, can be directly extracted from the linear branch of the interaction profile at large $h$. The value obtained from the curve is $80 \mathrm{fN}$. It corresponds to a $5.7 \mu \mathrm{m}$ diameter sphere having an apparent density of $1.08 \mathrm{~g} / \mathrm{cm}^{3}$, which is bigger than the expected value for polystyrene latex $\left(1.05 \mathrm{~g} / \mathrm{cm}^{3}\right)$. This discrepancy is due to the fact that the optical trap, used to prevent the lateral movement of a particle, exerts a light pressure on the sphere, which makes it apparently heavier. The decay length obtained by exponential regression from the potential profile at small $h$ is $13.6 \mathrm{~nm}$. It agrees well with the Debye screening length of $\kappa^{-1}=13.0 \mathrm{~nm}$ corresponding to the electrolyte concentration of $0.55 \mathrm{mM}$ used in the measurement.

Open symbols in Figure 5a show the interaction profiles between the sphere and the wall in the presence of dextran, of which the concentrations are indicated in the figure caption. Upon increasing the polymer concentration, no significant difference in the profiles could be detected. This is due to the fact that the equilibrium separation distance in the absence of polymer, $h_{\min }^{0}$, under these experimental conditions does not intersect with the range of the depletion-induced attraction. For dextran used in the experiment $\left\langle R_{\mathrm{g}}\right\rangle_{z}=43.7 \mathrm{~nm}$, which corresponds to a range of attraction of $2 \delta \approx 100 \mathrm{~nm}$. This range 


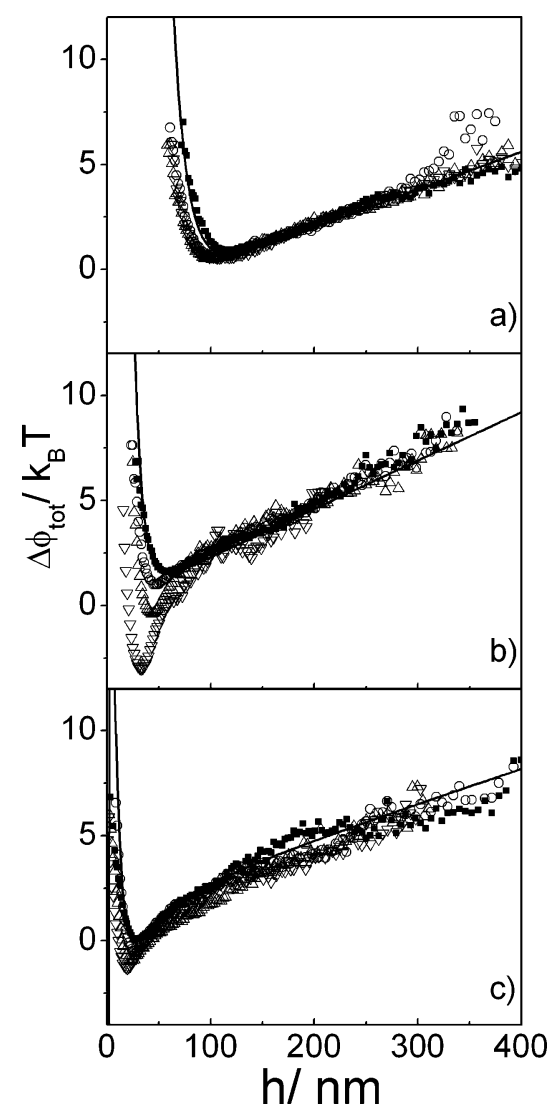

Figure 5. Interaction potential, $\Delta \phi_{\mathrm{tot}}(h)$, between a $5.7 \mu \mathrm{m}$ diameter PS sphere and a glass wall. Solid squares show the interaction profile in the absence of a polymer. Open symbols refer to the solutions with dextan $\left(\left\langle R_{\mathrm{g}}\right\rangle_{z}=43.7 \mathrm{~nm}\right)$, of which the concentrations are $(\mathrm{O})$ $1.4 \mathrm{~g} / \mathrm{L},(\triangle) 2.8 \mathrm{~g} / \mathrm{L}$, and $(\nabla) 3.6 \mathrm{~g} / \mathrm{L}$. The Debye length, $\kappa^{-1}$, is (a) $13 \mathrm{~nm}$, (b) $7.4 \mathrm{~nm}$, and (c) $5.6 \mathrm{~nm}$. The solid curves are the best fits in plots (a) and (b) according to eq 20; and in plot (c) the solid line represents a model calculation according to eq 20 including the estimated van der Waals attraction from eq 21.

is smaller than $h_{\min }^{0}=116 \mathrm{~nm}$. Hence, the depletion interaction is not measurable with TIRM under these particular experimental conditions. This finding is in agreement with the work of Pagac et al, ${ }^{19}$ where no significant attraction was found between a silica sphere and a glass wall in the presence of PEO. Also there, the sphere's equilibrium distance from the surface was larger than two depletion thicknesses.

In Figure $5 \mathrm{~b}$ we present interaction potentials, $\Delta \phi_{\text {tot }}(h)$, measured between a particle with the same diameter of $5.7 \mu \mathrm{m}$ and a glass surface in a solution with an increased electrolyte concentration of $1.6 \mathrm{mM}\left(\kappa^{-1}=7.6 \mathrm{~nm}\right)$. The decreased Debye length results in an increased negative slope of the potential profile at small $h$ and a shift of $h_{\min }^{0}$ to $58 \mathrm{~nm}$. From the fit of the potential curve in the absence of dextran, presented by the solid squares, we obtain $G=105 \mathrm{fN}$ and $\kappa^{-1}=8.7 \mathrm{~nm}$. The obtained Debye screening length corresponds well to the value of $7.6 \mathrm{~nm}$ as calculated from the used electrolyte concentration and the increase of $G$ can be explained by variations of particle size and density when changing from one particle to another. After polymer is added, the total interaction potentials (open symbols) deviate from the profile in the absence of dextran. Their minima shift to smaller separation distances and the slope on the right side of the potential becomes steeper at intermediate distances with increasing polymer concentration, because dextran molecules exert a measurable depletion interaction between the particle and the glass surface under these conditions. The resulting attraction becomes stronger with increasing polymer concentration as predicted from eq 10 and the potential profiles deepen. The obtained experimental results qualitatively follow our expectation on the basis of the theoretical predictions, which were plotted in Figure 2.

More experimental results are presented in Figure $5 \mathrm{c}$ using the highest electrolyte concentration $(2.9 \mathrm{mM})$. Here $\kappa^{-1}=5.6$ $\mathrm{nm}$ and causes a further shift of $h_{\min }^{0}$ to even smaller distances. The equilibrium separation distance is now $h_{\min }^{0}=27 \mathrm{~nm}$. The interaction profile, $\Delta \phi_{\text {tot. }}(h)$, in the absence of dextran, presented by solid squares, shows a significant curvature in the attractive part. It was not possible to fit this potential with the superposition of a gravitational contribution and an electrostatic term. Because the particle's equilibrium location is very close to the wall for $\kappa^{-1}=5.6 \mathrm{~nm}$, we conjecture that van der Waals attraction is dominating the potential in this range. This fact was first experimentally verified by Suresh and Walz using TIRM. ${ }^{42,43}$ The solid curve in Figure 5c presents the estimated total potential including van der Waals attraction between the sphere and the wall, which accordingly to Israelachvili ${ }^{35}$ reads as

$$
\phi_{\mathrm{vdW}, \text { plate-sphere }}=-A_{\mathrm{H}} \frac{a}{6 h}
$$

Here we roughly estimated the Hamaker constant as $A_{\mathrm{H}} \approx 0.2$ $k_{\mathrm{B}} T$ to achieve the best match of the experimental potential. This value corresponds very well to $A_{\mathrm{H}}<0.5 k_{\mathrm{B}} T$ calculated by Bevan and Prieve ${ }^{44}$ for the glass and PS surfaces using Lifshitz theory and incorporating retardation and screening by the presence of ions in solution. The strong van der Waals forces clearly dominate the attractive part of the interaction potential. As presented in Figure $5 c$, the potential profiles are not extensively influenced by the presence of dextran in the solution. The depletion attraction is a minor contribution to $\phi_{\mathrm{tot}}(h)$ now. Therefore, it was not possible to extract the depletion potential under these conditions.

To summarize these observations, we experimentally verified our predictions (section II.A) for the conditions under which the depletion interaction is measurable with TIRM. They are the following:

(a) in the absence of polymer, the equilibrium separation distance between a particle and a wall has to be smaller than two depletion thicknesses and significantly larger than the range of van der Waals attraction: $h_{\mathrm{vdW}}<h_{\text {min }}^{0} \leq 2 \delta$;

(b) the magnitude of the depletion interaction should be larger than approximately $k_{\mathrm{B}} T$ to make a clear difference between the total interaction profiles obtained in the presence and in the absence of polymer.

During the measurements in solution with the Debye screening length of $7.4 \mathrm{~nm}$, shown in Figure 5b, we fulfilled these conditions.

IV.B. Depletion Potentials; Comparison with Theory. By subtracting the profile for zero dextran concentration from the potential profiles obtained in the presence of polymer, we obtained the pure depletion interaction potentials. These depletion curves are plotted as open symbols in Figure 6 for different polymer concentrations, $c_{\mathrm{p}}$.

In our system the depletion interaction between a particle and a wall presented in Figure 6 at separation distances smaller than two depletion thicknesses is purely attractive. Contrary to earlier reports ${ }^{20}$ we did not find any repulsive contribution to the depletion interaction, which is agreement with careful simulations of colloid polymer mixtures. ${ }^{45}$ At larger distances the potential goes to

(42) Suresh, L.; Walz, J. Y. J. Colloid Interface Sci. 1996, 183, 199-213.

(43) Suresh, L.; Walz, J. Y. J. Colloid Interface Sci. 1997, 196, 177-190.

(44) Bevan, M. A.; Prieve, D. C. Langmuir 1999, 15, 7925-7936.

(45) Bolhuis, P. G.; Louis, A. A.; Hansen, J. P.; Meijer, E. J. J. Chem. Phys 2001, 114, 4296-4311. 


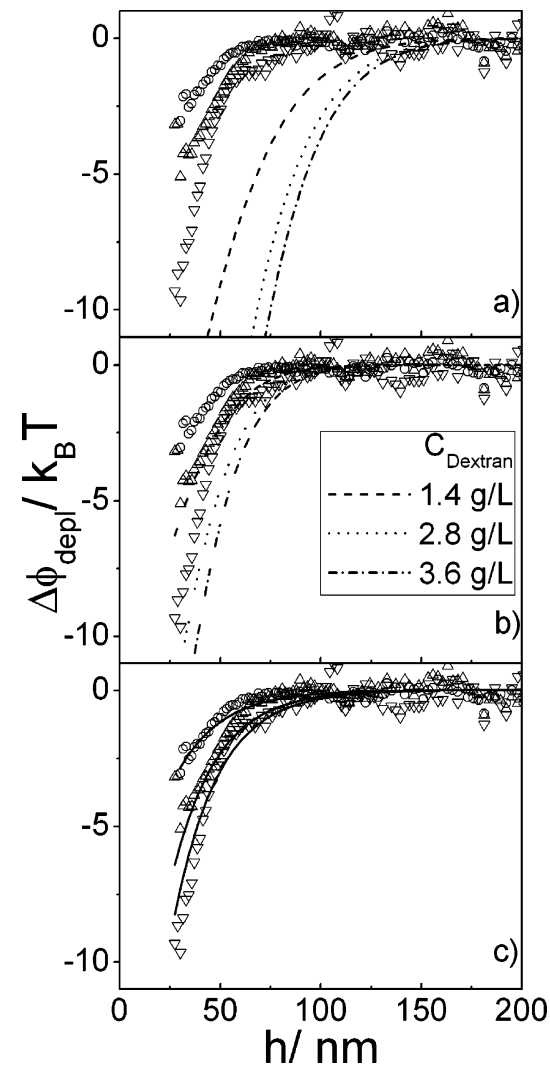

Figure 6. Depletion potential between a $5.7 \mu \mathrm{m}$ diameter polystyrene sphere and a glass wall in aqueous solution with dextran, of which the concentrations are $(\bigcirc) 1.4 \mathrm{~g} / \mathrm{L},(\triangle) 2.8 \mathrm{~g} / \mathrm{L}$, and $(\nabla) 3.6 \mathrm{~g} / \mathrm{L}$. In all solutions $\kappa^{-1}=7.4 \mathrm{~nm}$. The curves are (a) model calculations using eq 10 for a monodisperse polymer with $\left\langle R_{\mathrm{g}}\right\rangle_{z}=43.7 \mathrm{~nm}$ and $M_{\mathrm{w}}=2.7 \times 10^{6} \mathrm{~g} / \mathrm{mol}$, (b) model calculations using eq 10 for a polydisperse polymer with $\left\langle R_{\mathrm{g}}\right\rangle_{\mathrm{w}}=30.9 \mathrm{~nm}$ and $M_{\mathrm{w}}=2.7 \times 10^{6}$ $\mathrm{g} / \mathrm{mol}$, and (c) nonlinear least-squares fits with eq 17 using the full molar mass distribution of the polymer.

zero as expected from the theoretical predictions (eq 17). On the basis of the PHS approximation for monodisperse polymers, eq 10 predicts a stronger depletion attraction with increasing macromolecule concentration; this trend is seen in Figure 6. However, there is no quantitative agreement between the experimental profiles and this simple theory. The dashed lines in Figure 6a were calculated from eq 10 using the $z$-averaged radius of gyration of dextran, $\left\langle R_{\mathrm{g}}\right\rangle_{z}=43.7 \mathrm{~nm}$, its weight averaged molar mass, $M_{\mathrm{W}}=2.7 \times 10^{6} \mathrm{~g} / \mathrm{mol}$, and overlap concentration, $c^{*}=12.4 \mathrm{~g} / \mathrm{L}$. Here the particle radius, $a=2.85 \mu \mathrm{m}$ was kept as in the experiment. It is clear that the very simple PHS model significantly overestimates the range and depth of the depletion potential. This is due to the fact that the dextran used in the experiment was highly polydisperse and cannot be described as a monodisperse chain with $R_{\mathrm{g}}=43.7 \mathrm{~nm}$.

To take into account the polydispersity of dextran, first we simply substituted $R_{\mathrm{g}}$ in eq 10 with the weight-average radius of gyration $\left\langle R_{\mathrm{g}}\right\rangle_{\mathrm{w}}$. The latter depends on the molar mass distribution of dextran $\Psi(M)$ and follows from

$$
\left\langle R_{\mathrm{g}}\right\rangle_{\mathrm{w}}=\frac{\int_{0}^{\infty} \Psi(M) b \mathrm{~d} M}{\int_{0}^{\infty} \Psi(M) M^{-1 / 2} \mathrm{~d} M}
$$

Here the pre-factor $b$, as in eq 16, depends on the segment length and the chain architecture and can be estimated by using the expression for the $z$-averaged radius of gyration:

$$
\left\langle R_{\mathrm{g}}\right\rangle_{z}=\frac{\int_{0}^{\infty} \Psi(M) M^{1 / 2} b \mathrm{~d} M}{\int_{0}^{\infty} \Psi(M) \mathrm{d} M}
$$

Because we measured $\left\langle R_{\mathrm{g}}\right\rangle_{z}$ and $\Psi(M)$ is known, it was possible to determine $b$ from eq 23 . In this way we found $\left\langle R_{\mathrm{g}}\right\rangle_{\mathrm{w}}=30.9$ $\mathrm{nm}$ for dextran. Dashed lines in Figure $6 \mathrm{~b}$ are calculated from eq 10 after substitution of $R_{\mathrm{g}}$ with $\left\langle R_{\mathrm{g}}\right\rangle_{\mathrm{w}}$. This replacement provides a better match to the experimental data than calculations done using the $z$-averaged radius of gyration. Nevertheless, the depletion potential range and depth are still predicted too large.

Finally, we compare the experimentally measured depletion potentials with the full result of eq 17 for the depletion interaction due to polydisperse ideal polymers using the entire molar mass distribution of dextran, $\Psi(M)$. We used eq 16, which is the scaling law relating the molar mass, $M$, and the radius of gyration of polymer, $R_{\mathrm{g}}$, to obtain the distribution of the radii of gyration. The square root in this equation corresponds to the ideal polymer chain regime in a $\theta$-solvent. It was shown by Koning et al. ${ }^{46}$ that water is very close to a $\theta$-solvent for dextran. Therefore, using $R_{\mathrm{g}}=b \sqrt{M}$ is justified for our calculations. The distribution of the radii of gyration of dextran is required in eqs 13 and 14 to calculate the polydisperse depletion thickness, $\delta_{\text {poly }}$, and the polydisperse partition coefficient, $\chi_{\text {poly }}$. Consequently, given the molar mass distribution of polymer, $\Psi(M)$, the pre-factor $b$, which depends on the segment length and the polymer chain architecture, is the only adjustable parameter required to describe the experimental depletion potentials. Qualitatively one would expect the contact value of the potential and its range to increase with increasing $b$ and vice versa. However, for a quantitative prediction, molecular simulations would be required. We used a global nonlinear least squares algorithm to simultaneously fit the curves from all dextran concentrations. The results are presented as full curves in Figure 6c. A good agreement between the theory and experiment has been achieved now. The magnitude of $b$ obtained from the fit procedure is $0.24 \mathrm{~nm} \cdot \mathrm{kg}^{-1 / 2} \cdot \mathrm{mol}^{-1 / 2}$, which lies between the data of Nordmeier ${ }^{47}$ and Ioan et al. ${ }^{48}$ Our analysis manifests that a satisfactory description of the depletion potential is not possible using averaged values of the radius of gyration. For polymers with a broad molar mass distribution the full distribution has to be incorporated into the theoretical expressions for $\phi_{\text {depl }}(h)$. This is well in line with the theoretical work of Tuinier and Petukhov, ${ }^{31}$ who showed for a plate-plate geometry that the depletion potential due to polydisperse depletants cannot be described satisfactorily using an averaged value of $R_{\mathrm{g}}$ if the molar mass distribution has a standard deviation larger than 0.7 of the center value.

\section{Conclusions}

We measured the depletion potential between a sphere and a wall in a solution of polydisperse dextran with total internal reflection microscopy. It was shown theoretically and experimentally verified under which conditions the depletion interaction should be measurable by TIRM. We found that the particle equilibrium distance from the surface in the absence of polymer has to be shorter than twice the depletion thickness and longer than the range of the van der Waals forces: $h_{\mathrm{vdW}}<h_{\min }^{0} \leq 2 \delta$. This finding explains some of the contradictions in the literature about experimentally determined depletion potentials.

(46) Koning, M. M. G.; van Eedenburg, J.; de Bruijne, D. W. In Food Colloids and Polymers; Dickinson, E., Walstra, P., Eds.; Royal Sosiety of Chemistry: Cambridge, U.K., 1993; p 103.

(47) Nordmeier, E. J. Phys. Chem. 1993, 97, 5770-5785. 5739 .

(48) Ioan, C. E.; Aberle, T.; Burchard, W. Macromolecules 2000, 33, 5730- 
At the same time the magnitude of depletion attraction must be larger than approximately $k_{\mathrm{B}} T$ to achieve a clear difference between the total interaction profiles obtained in the presence and in the absence of a polymer. By varying the solution Debye screening length, one can adjust $h_{\min }{ }^{0}$ to the desired value, and upon changing the polymer concentration, one can adjust the magnitude of the depletion attraction in the required way.

Further, we investigated the influence of the polymer mass distribution on the depletion potential. For the widely applied dextran with broad distribution, we show that the depletion potential cannot be predicted using average values for $R_{\mathrm{g}}$. Rather the full expression for the molar mass distribution has to be incorporated into the equation for the calculation of the polymer partition coefficient and the depletion thickness to achieve a reasonable description of the depletion potential.

Acknowledgment. We thank Professor Cohen Stuart for useful suggestions.

LA061657M 